\title{
On a coupled system of fractional differential equations with nonlocal non-separated boundary conditions
}

\author{
Sabbavarapu Nageswara Rao ${ }^{1 *}$ and Meshari Alesemi ${ }^{1}$
}

\section{"Correspondence:}

snrao@jazanu.edu.sa

'Department of Mathematics,

Faculty of Science, Jazan University, Jazan, Saudi Arabia

\begin{abstract}
We solve a coupled system of nonlinear fractional differential equations equipped with coupled fractional nonlocal non-separated boundary conditions by using the Banach contraction principle and the Leray-Schauder fixed point theorem. Finally, we give examples to demonstrate our results.
\end{abstract}

MSC: 34A08; 34B15

Keywords: Caputo fractional derivative; Nonlocal; Non-separated boundary conditions; Existence of solution

\section{Introduction}

In last few years, some physical phenomena were described through fractional differential equations and compared with integer order differential equations which have better results, which is why researchers of different areas have paid great attention to study fractional differential equation. Fractional differential equations arise in the mathematical modeling of systems and processes occurring in many engineering and scientific disciplines such as physics, chemistry, polymer rheology, control theory, diffusive transport akin to diffusion, electrical networks, probability, etc. For details, see [14-16, 22, 23, 27, $29,37]$. In the last few decades, fractional-order differential equations equipped with a variety of boundary conditions have been studied. The literature on the topic includes the existence and uniqueness results related to classical, periodic/anti-periodic, nonlocal, multi-point, and integral boundary conditions; for instance, see $[1,3,6,7,9,11,17,19,24$, $26,28,30,34,36]$ and the references therein.

The existence and uniqueness of positive solutions for such problems have become an important area of investigation in recent years. Ahmad and Nieto [6] investigated the existence and uniqueness of solutions for an anti-periodic fractional boundary value problem

$$
\begin{aligned}
& { }^{c} D^{q} x(t)=f(t, x(t)), \quad t \in[0, T], 1<q \leq 2, T>0, \\
& x(0)=-x(T), \quad{ }^{c} D^{p} x(0)=-{ }^{c} D^{p} x(T), \quad 0<p<1,
\end{aligned}
$$

where ${ }^{c} D^{q}$ denotes the Caputo fractional derivative of order $q, f$ is a given continuous function.

(c) The Author(s) 2019. This article is distributed under the terms of the Creative Commons Attribution 4.0 International License (http://creativecommons.org/licenses/by/4.0/), which permits unrestricted use, distribution, and reproduction in any medium, provided you give appropriate credit to the original author(s) and the source, provide a link to the Creative Commons license, and indicate if changes were made. 
Liu and Liu [24] investigated the existence and uniqueness of solutions for fractional differential equations with fractional non-separated boundary conditions in the form of

$$
\begin{aligned}
& { }^{c} D^{\alpha} x(t)=f(t, x(t)), \quad t \in[0, T], 1<\alpha \leq 2, T>0, \\
& a_{1} x(0)+b_{1} x(T)=c_{1}, \quad a_{2}\left({ }^{c} D^{\gamma} x(0)\right)+b_{2}\left({ }^{c} D^{\gamma} x(T)\right)=c_{2}, \quad 0<\gamma<1,
\end{aligned}
$$

where ${ }^{c} D^{\alpha}$ denotes the Caputo fractional derivative of order $\alpha, f$ is a continuous function on $[0, T] \times \mathbb{R}$ and $a_{i}, b_{i}, c_{i}, i=1,2$ are real constants such that $a_{1}+b_{1} \neq 0$ and $b_{2} \neq 0$.

The system of fractional differential equations boundary value problems has also received much attention and its research has developed very rapidly; see $[2,4,5,8,10,12$, $13,20,21,25,31-33,35]$. Recently, Alsulalt et al. [13] established the existence and uniqueness results for a nonlinear coupled system of Caputo type fractional differential equations supplemented with non-separated coupled boundary conditions.

In this paper, motivated by the aforementioned work, we consider the existence and uniqueness of solutions for a coupled system of fractional differential equation

$$
\begin{cases}{ }^{c} D_{0^{+}}^{\alpha} u(t)=f(t, u(t), v(t)), & 0<t<1, \\ { }^{c} D_{0^{+}}^{\beta} \nu(t)=g(t, u(t), v(t)), & 0<t<1,\end{cases}
$$

subject to the fractional non-separated coupled boundary conditions

$$
\left\{\begin{array}{lll}
u(0)=\lambda_{1} v(1), & { }^{c} D_{0^{+}}^{\gamma} u(1)=\lambda_{2}{ }^{c} D_{0^{+}}^{\gamma} v(\xi), & 0<\gamma<1, \\
v(0)=\mu_{1} u(1), & { }^{c} D_{0^{+}}^{\gamma} \nu(1)=\mu_{2}{ }^{c} D_{0^{+}}^{\gamma} u(\xi), & 0<\gamma<1,
\end{array}\right.
$$

where $\alpha, \beta \in(1,2], \xi \in(0,1),{ }^{c} D_{0^{+}}^{\alpha}$ and ${ }^{c} D_{0^{+}}^{\beta}$ are the Caputo fractional derivatives of order $\alpha$ and $\beta$, respectively, $f, g \in C([0,1] \times[0,+\infty) \times[0,+\infty),[0,+\infty))$ and $\lambda_{i}, \mu_{i}, i=1,2$ real constants with $\mu_{1} \lambda_{1} \neq 1$ and $\mu_{2} \lambda_{2} \xi^{2(1-\gamma)} \neq 1$.

This paper is organized as follows. In Sect. 2, we recall some basic definitions of fractional calculus and present an auxiliary lemma, which plays a major role in obtaining the main result. In Sect. 3, we established the existence and uniqueness results for a nonlinear coupled system of fractional differential equation (1)-(2). Finally, as an application, we give two examples to illustrate our results.

\section{Preliminaries}

Let us now recall some basic definitions of fractional derivative [37] and prove a lemma before stating our main results.

Definition 2.1 The fractional integral of order $q$ with the lower limit zero for a function $f:[0, \infty) \rightarrow \mathbb{R}$ is defined as

$$
I^{q} f(t)=\frac{1}{\Gamma(q)} \int_{0}^{t} \frac{f(s)}{(t-s)^{1-q}} d s, \quad t>0, q>0,
$$

provided the right hand side is point-wise defined on $[0, \infty)$, where $\Gamma(\cdot)$ is the gamma function, which is defined by $\Gamma(q)=\int_{0}^{\infty} t^{q-1} e^{-t} d t$. 
Definition 2.2 The Riemann-Liouville fractional derivative of order $q>0, n-1<q<n$, $n \in \mathbb{N}$, is defined as

$$
D_{0^{+}}^{q} f(t)=\frac{1}{\Gamma(n-q)}\left(\frac{d}{d t}\right)^{n} \int_{0}^{t}(t-s)^{n-q-1} f(s) d s
$$

where the function $f(t)$ has an absolutely continuous derivative up to order $(n-1)$.

Definition 2.3 The Caputo derivative of order $q$ for a function $f:[0, \infty) \rightarrow \mathbb{R}$ with $f(t) \in$ $C^{n}[0, \infty)$ is defined by

$$
{ }^{c} D^{q} f(t)=\frac{1}{\Gamma(n-q)} \int_{0}^{t} \frac{f^{(n)}(s)}{(t-s)^{q+1-n}} d s=I^{n-q} f^{(n)}(t), \quad t>0, n-1<q<n .
$$

Furthermore, we noted that the Riemann-Liouville fractional derivative of a constant is usually nonzero which can cause serious problems in real world applications. We have

$$
\begin{aligned}
{ }^{c} D^{q} f(t) & =\frac{1}{\Gamma(n-q)} \int_{0}^{t} \frac{f^{n}(s)}{(t-s)^{q+1-n}} d s \\
& =D^{q} f(t)-\sum_{k=0}^{n-1} \frac{f^{(k)}(0)}{\Gamma(k-q+1)} t^{k-q} \\
& =D^{q}\left[f(t)-\sum_{k=0}^{n-1} \frac{f^{(k)}(0)}{k !} t^{k}\right], \quad t>0, n-1<q<n .
\end{aligned}
$$

So, we preferred to use Caputo's definition which gives better results than those of Riemann-Liouville.

Lemma 2.1 Let $\Delta=1-\lambda_{2} \mu_{2} \xi^{2(1-\gamma)} \neq 0$ and $\lambda_{1} \mu_{1} \neq 1$. Let $\phi, \psi \in C([0,1], \mathbb{R})$. Then the solution of the linear fractional differential equations:

$$
\begin{cases}{ }^{c} D_{0^{+}}^{\alpha} u(t)=\phi(t), & t \in[0,1], 1<\alpha \leq 2, \\ { }^{c} D_{0^{+}}^{\beta} \nu(t)=\psi(t), & t \in[0,1], 1<\beta \leq 2,\end{cases}
$$

supplemented with the boundary conditions (2) is given by

$$
\begin{aligned}
u(t)= & \frac{\mu_{2} \Gamma(2-\gamma)}{\Delta}\left[\frac{\lambda_{1}\left(\mu_{1} \lambda_{2} \xi^{1-\gamma}+1\right)}{1-\lambda_{1} \mu_{1}}+\lambda_{2} \xi^{1-\gamma} t\right] A_{3} \\
& -\frac{\Gamma(2-\gamma)}{\Delta}\left[\frac{\lambda_{1}\left(\mu_{1} \lambda_{2} \xi^{1-\gamma}+1\right)}{1-\lambda_{1} \mu_{1}}+\lambda_{2} \xi^{1-\gamma} t\right] B_{3} \\
& +\frac{\lambda_{2} \Gamma(2-\gamma)}{\Delta}\left[\frac{\lambda_{1}\left(\mu_{1}+\mu_{2} \xi^{1-\gamma}\right)}{1-\lambda_{1} \mu_{1}}+t\right] B_{2} \\
& -\frac{\Gamma(2-\gamma)}{\Delta}\left[\frac{\lambda_{1}\left(\mu_{1}+\mu_{2} \xi^{1-\gamma}\right)}{1-\lambda_{1} \mu_{1}}+t\right] A_{2} \\
& +\frac{\lambda_{1}}{1-\mu_{1} \lambda_{1}}\left(\mu_{1} A_{1}+B_{1}\right)+\int_{0}^{t} \frac{(t-s)^{\alpha-1}}{\Gamma(\alpha)} \phi(s) d s
\end{aligned}
$$


and

$$
\begin{aligned}
v(t)= & \frac{\mu_{2} \Gamma(2-\gamma)}{\Delta}\left[\frac{\mu_{1}\left(\lambda_{1}+\lambda_{2} \xi^{1-\gamma}\right)}{1-\lambda_{1} \mu_{1}}+t\right] A_{3} \\
& -\frac{\Gamma(2-\gamma)}{\Delta}\left[\frac{\mu_{1}\left(\lambda_{1}+\lambda_{2} \xi^{1-\gamma}\right)}{1-\lambda_{1} \mu_{1}}+t\right] B_{3} \\
& +\frac{\lambda_{2} \Gamma(2-\gamma)}{\Delta}\left[\frac{\mu_{1}\left(\lambda_{1} \mu_{2} \xi^{1-\gamma}+1\right)}{1-\lambda_{1} \mu_{1}}+\mu_{2} \xi^{1-\gamma} t\right] B_{2} \\
& -\frac{\Gamma(2-\gamma)}{\Delta}\left[\frac{\mu_{1}\left(\lambda_{1} \mu_{2} \xi^{1-\gamma}+1\right)}{1-\lambda_{1} \mu_{1}}+\mu_{2} \xi^{1-\gamma} t\right] A_{2} \\
& +\frac{\mu_{1}}{1-\mu_{1} \lambda_{1}}\left(A_{1}+\lambda_{1} B_{1}\right)+\int_{0}^{t} \frac{(t-s)^{\beta-1}}{\Gamma(\beta)} \psi(s) d s
\end{aligned}
$$

where

$$
\begin{array}{ll}
A_{1}=\int_{0}^{1} \frac{(1-s)^{\alpha-1}}{\Gamma(\alpha)} \phi(s) d s, & B_{1}=\int_{0}^{1} \frac{(1-s)^{\beta-1}}{\Gamma(\beta)} \psi(s) d s, \\
A_{2}=\int_{0}^{1} \frac{(1-s)^{\alpha-\gamma-1}}{\Gamma(\alpha-\gamma)} \phi(s) d s, & B_{2}=\int_{0}^{\xi} \frac{(\xi-s)^{\beta-\gamma-1}}{\Gamma(\beta-\gamma)} \psi(s) d s, \\
A_{3}=\int_{0}^{\xi} \frac{(\xi-s)^{\alpha-\gamma-1}}{\Gamma(\alpha-\gamma)} \phi(s) d s, & B_{3}=\int_{0}^{1} \frac{(1-s)^{\beta-\gamma-1}}{\Gamma(\beta-\gamma)} \psi(s) d s .
\end{array}
$$

Proof It is well know [22] that the general solution of the fractional differential equations in (3) can be written by

$$
\begin{aligned}
& u(t)=c_{0}+c_{1} t+\int_{0}^{t} \frac{(t-s)^{\alpha-1}}{\Gamma(\alpha)} \phi(s) d s, \\
& v(t)=d_{0}+d_{1} t+\int_{0}^{t} \frac{(t-s)^{\beta-1}}{\Gamma(\beta)} \psi(s) d s,
\end{aligned}
$$

where $c_{i}, d_{i}, i=0,1$ are arbitrary constants. Since

$$
{ }^{c} D^{\gamma} k=0 \quad(k \text { is a constant }), \quad{ }^{c} D^{\gamma} t=\frac{t^{1-\gamma}}{\Gamma(2-\gamma)}, \quad{ }^{c} D^{\gamma} I^{\alpha} y(t)=I^{\alpha-\gamma} y(t),
$$

from (6) and (7), we have

$$
\begin{aligned}
& { }^{c} D^{\gamma} u(t)=c_{1} \frac{t^{1-\gamma}}{\Gamma(2-\gamma)}+\int_{0}^{t} \frac{(t-s)^{\alpha-\gamma-1}}{\Gamma(\alpha-\gamma)} \phi(s) d s, \\
& { }^{c} D^{\gamma} \nu(t)=d_{1} \frac{t^{1-\gamma}}{\Gamma(2-\gamma)}+\int_{0}^{t} \frac{(t-s)^{\beta-\gamma-1}}{\Gamma(\beta-\gamma)} \psi(s) d s .
\end{aligned}
$$

Using the boundary conditions in (6), we have

$$
\begin{aligned}
& u(0)=\lambda_{1} v(1) \quad \Rightarrow \quad c_{0}=\lambda_{1}\left(d_{0}+d_{1}+B_{1}\right), \\
& v(0)=\mu_{1} u(1) \quad \Rightarrow \quad d_{0}=\mu_{1}\left(c_{0}+c_{1}+A_{1}\right) .
\end{aligned}
$$


Using the boundary conditions in (7), we have

$$
\begin{array}{lll}
{ }^{c} D^{\gamma} u(1)=\lambda_{2}{ }^{c} D^{\gamma} v(\xi) & \Rightarrow & c_{1}=\lambda_{2} \xi^{1-\gamma} d_{1}+\Gamma(2-\gamma)\left[\lambda_{2} B_{2}-A_{2}\right] \\
{ }^{c} D^{\gamma} v(1)=\mu_{2}{ }^{c} D^{\gamma} u(\xi) & \Rightarrow & d_{1}=\mu_{2} \xi^{1-\gamma} c_{1}-\Gamma(2-\gamma)\left[\mu_{2} A_{3}-B_{3}\right] .
\end{array}
$$

From the last two relations we find

$$
\begin{aligned}
& c_{1}=\frac{\Gamma(2-\gamma)}{\Delta}\left[\lambda_{2} \mu_{2} \xi^{1-\gamma} A_{3}-\lambda_{2} \xi^{1-\gamma} B_{3}+\lambda_{2} B_{2}-A_{2}\right] \\
& d_{1}=\frac{\Gamma(2-\gamma)}{\Delta}\left[\mu_{2} A_{3}-B_{3}-\mu_{2} \xi^{1-\gamma} A_{2}+\mu_{2} \lambda_{2} \xi^{1-\gamma} B_{2}\right] .
\end{aligned}
$$

Substituting $c_{1}$ and $d_{1}$ in the first two relations, we find

$$
\begin{aligned}
c_{0}= & \frac{\lambda_{1}}{1-\lambda_{1} \mu_{1}}\left[\mu_{1} c_{1}+\mu_{1} A_{1}+d_{1}+B_{1}\right] \\
= & \frac{\lambda_{1}}{1-\lambda_{1} \mu_{1}}\left[\frac{\Gamma(2-\gamma) \mu_{2}\left(\mu_{1} \lambda_{2} \xi^{1-\gamma}+1\right)}{\Delta} A_{3}-\frac{\Gamma(2-\gamma)\left(\mu_{1} \lambda_{2} \xi^{1-\gamma}+1\right)}{\Delta} B_{3}\right. \\
& \left.-\frac{\Gamma(2-\gamma)\left(\mu_{1}+\mu_{2} \xi^{1-\gamma}\right)}{\Delta} A_{2}+\frac{\Gamma(2-\gamma) \lambda_{2}\left(\mu_{1}+\mu_{2} \xi^{1-\gamma}\right)}{\Delta} B_{2}+\mu_{1} A_{1}+B_{1}\right]
\end{aligned}
$$

and

$$
\begin{aligned}
d_{0}= & \frac{\mu_{1}}{1-\lambda_{1} \mu_{1}}\left[\lambda_{1} d_{1}+\lambda_{1} B_{1}+c_{1}+A_{1}\right] \\
= & \frac{\mu_{1}}{1-\lambda_{1} \mu_{1}}\left[\frac{\Gamma(2-\gamma) \mu_{2}\left(\lambda_{1}+\lambda_{2} \xi^{1-\gamma}\right)}{\Delta} A_{3}-\frac{\Gamma(2-\gamma)\left(\lambda_{1}+\lambda_{2} \xi^{1-\gamma}\right)}{\Delta} B_{3}\right. \\
& \left.-\frac{\Gamma(2-\gamma)\left(\lambda_{1} \mu_{2} \xi^{1-\gamma}+1\right)}{\Delta} A_{2}+\frac{\Gamma(2-\gamma) \lambda_{2}\left(\mu_{2} \lambda_{1} \xi^{1-\gamma}+1\right)}{\Delta} B_{2}+\lambda_{1} B_{1}+A_{1}\right] .
\end{aligned}
$$

Inserting the values of $c_{i}, d_{i}, i=0,1$ in (6) and (7), we get solutions (4) and (5). The converse of the above proof is as follows.

For any $t \in[0,1]$, taking the $\gamma$-fractional derivative for (4) and (5) yields

$$
\begin{aligned}
{ }^{c} D^{\gamma} u(t)= & \frac{t^{1-\gamma}}{\Delta}\left[\mu_{2} \lambda_{2} \xi^{1-\gamma} A_{3}-\lambda_{2} \xi^{1-\gamma} B_{3}+\lambda_{2} B_{2}-A_{2}\right] \\
& +\int_{0}^{t} \frac{(t-s)^{\alpha-\gamma-1}}{\Gamma(\alpha-\gamma)} \phi(s) d s
\end{aligned}
$$

and

$$
\begin{aligned}
{ }^{c} D^{\gamma} \nu(t)= & \frac{t^{1-\gamma}}{\Delta}\left[\mu_{2} A_{3}-B_{3}+\lambda_{2} \mu_{2} \xi^{1-\gamma} B_{2}-\mu_{2} \xi^{1-\gamma} A_{2}\right] \\
& +\int_{0}^{t} \frac{(t-s)^{\beta-\gamma-1}}{\Gamma(\beta-\gamma)} \psi(s) d s .
\end{aligned}
$$

Checking the first boundary condition, we see that

$$
u(0)=\lambda_{1} v(1), \quad{ }^{c} D_{0^{+}}^{\gamma} u(1)=\lambda_{2}{ }^{c} D_{0^{+}}^{\gamma} \nu(\xi) .
$$


Moreover, in checking the second boundary condition we get

$$
v(0)=\mu_{1} u(1), \quad{ }^{c} D_{0^{+}}^{\gamma} \nu(1)=\mu_{2}{ }^{c} D_{0^{+}}^{\gamma} u(\xi) .
$$

Taking the $\alpha$-fractional derivative and $\beta$-fractional derivative yields

$$
{ }^{c} D_{0^{+}}^{\alpha} u(t)=\phi(t) ; \quad{ }^{c} D_{0^{+}}^{\beta} \nu(t)=\psi(t)
$$

which is what we set out to prove.

\section{Main results}

Let $X=\{u(t) \mid u(t) \in C([0,1], \mathbb{R})\}$ denote the Banach space of all continuous functions from $[0,1]$ into $\mathbb{R}$ equipped with the norm $\|u\|=\sup \{|u(t)|, t \in[0,1]\}$. Obviously, $(X,\|\cdot\|)$ is a Banach space. Then the product space $(X \times X,\|(u, v)\|)$ is also a Banach space equipped with the norm $\|(u, v)\|=\|u\|+\|v\|$.

In view of Lemma 2.1, we define the operator $Q: X \times X \rightarrow X \times X$ by

$$
Q(u, v)=\left(Q_{1}(u, v), Q_{2}(u, v)\right) .
$$

Here

$$
\begin{aligned}
Q_{1}(u, v)(t)= & \frac{\mu_{2} \Gamma(2-\gamma)}{\Delta}\left[\frac{\lambda_{1}\left(\mu_{1} \lambda_{2} \xi^{1-\gamma}+1\right)}{1-\lambda_{1} \mu_{1}}+\lambda_{2} \xi^{1-\gamma} t\right] A_{3 f} \\
& -\frac{\Gamma(2-\gamma)}{\Delta}\left[\frac{\lambda_{1}\left(\mu_{1} \lambda_{2} \xi^{1-\gamma}+1\right)}{1-\lambda_{1} \mu_{1}}+\lambda_{2} \xi^{1-\gamma} t\right] B_{3 g} \\
& -\frac{\Gamma(2-\gamma)}{\Delta}\left[\frac{\lambda_{1}\left(\mu_{1}+\mu_{2} \xi^{1-\gamma}\right)}{1-\lambda_{1} \mu_{1}}+t\right] A_{2 f} \\
& +\frac{\lambda_{2} \Gamma(2-\gamma)}{\Delta}\left[\frac{\lambda_{1}\left(\mu_{1}+\mu_{2} \xi^{1-\gamma}\right)}{1-\lambda_{1} \mu_{1}}+t\right] B_{2 g} \\
& +\frac{\lambda_{1}}{1-\mu_{1} \lambda_{1}}\left(\mu_{1} A_{1 f}+B_{1 g}\right)+\int_{0}^{t} \frac{(t-s)^{\alpha-1}}{\Gamma(\alpha)} f(s, u(s), v(s)) d s
\end{aligned}
$$

and

$$
\begin{aligned}
Q_{2}(u, v)(t)= & \frac{\mu_{2} \Gamma(2-\gamma)}{\Delta}\left[\frac{\mu_{1}\left(\lambda_{1}+\lambda_{2} \xi^{1-\gamma}\right)}{1-\lambda_{1} \mu_{1}}+t\right] A_{3 f} \\
& -\frac{\Gamma(2-\gamma)}{\Delta}\left[\frac{\mu_{1}\left(\lambda_{1}+\lambda_{2} \xi^{1-\gamma}\right)}{1-\lambda_{1} \mu_{1}}+t\right] B_{3 g} \\
& -\frac{\Gamma(2-\gamma)}{\Delta}\left[\frac{\mu_{1}\left(\lambda_{1} \mu_{2} \xi^{1-\gamma}+1\right)}{1-\lambda_{1} \mu_{1}}+\mu_{2} \xi^{1-\gamma} t\right] A_{2 f} \\
& +\frac{\lambda_{2} \Gamma(2-\gamma)}{\Delta}\left[\frac{\mu_{1}\left(\lambda_{1} \mu_{2} \xi^{1-\gamma}+1\right)}{1-\lambda_{1} \mu_{1}}+\mu_{2} \xi^{1-\gamma} t\right] B_{2 g} \\
& +\frac{\mu_{1}}{1-\mu_{1} \lambda_{1}}\left(\lambda_{1} B_{1 g}+A_{1 f}\right)+\int_{0}^{t} \frac{(t-s)^{\beta-1}}{\Gamma(\beta)} g(s, u(s), v(s)) d s .
\end{aligned}
$$

Here

$$
A_{1 f}=\int_{0}^{1} \frac{(1-s)^{\alpha-1}}{\Gamma(\alpha)} f(s, u(s), v(s)) d s, \quad B_{1 g}=\int_{0}^{1} \frac{(1-s)^{\beta-1}}{\Gamma(\beta)} g(s, u(s), v(s)) d s,
$$




$$
\begin{array}{ll}
A_{2 f}=\int_{0}^{1} \frac{(1-s)^{\alpha-\gamma-1}}{\Gamma(\alpha-\gamma)} f(s, u(s), v(s)) d s, & B_{2 g}=\int_{0}^{\xi} \frac{(\xi-s)^{\beta-\gamma-1}}{\Gamma(\beta-\gamma)} g(s, u(s), v(s)) d s, \\
A_{3 f}=\int_{0}^{\xi} \frac{(\xi-s)^{\alpha-\gamma-1}}{\Gamma(\alpha-\gamma)} f(s, u(s), v(s)) d s, & B_{3 g}=\int_{0}^{1} \frac{(1-s)^{\beta-\gamma-1}}{\Gamma(\beta-\gamma)} g(s, u(s), v(s)) d s .
\end{array}
$$

We use the following notations for convenience:

$$
\begin{aligned}
\sigma_{1}= & \frac{\left|\mu_{2}\right| \Gamma(2-\gamma)}{\left|1-\lambda_{2} \mu_{2} \xi^{2(1-\gamma)}\right|}\left[\frac{\left|\lambda_{1}\right|\left(\left|\mu_{1}\right|\left|\lambda_{2}\right| \xi^{1-\gamma}+1\right)}{\left|1-\lambda_{1} \mu_{1}\right|}+\left|\lambda_{2}\right| \xi^{1-\gamma}\right] \frac{\xi^{\alpha-\gamma}}{\Gamma(\alpha-\gamma+1)} \\
& +\frac{\Gamma(2-\gamma)}{\left|1-\lambda_{2} \mu_{2} \xi^{2(1-\gamma)}\right|}\left[\frac{\left|\lambda_{1}\right|\left(\left|\mu_{1}\right|+\left|\mu_{2}\right| \xi^{1-\gamma}\right)}{\left|1-\lambda_{1} \mu_{1}\right|}+1\right] \frac{1}{\Gamma(\alpha-\gamma+1)} \\
& +\left[\frac{\left|\lambda_{1}\right|\left|\mu_{1}\right|}{\left|1-\mu_{1} \lambda_{1}\right|}+1\right] \frac{1}{\Gamma(\alpha+1)}, \\
\sigma_{2}= & \frac{\left|\lambda_{2}\right| \Gamma(2-\gamma)}{\left|1-\lambda_{2} \mu_{2} \xi^{2(1-\gamma)}\right|}\left[\frac{\left|\lambda_{1}\right|\left(\left|\mu_{1}\right|+\left|\mu_{2}\right| \xi^{1-\gamma}\right)}{\left|1-\lambda_{1} \mu_{1}\right|}+1\right] \frac{\xi^{\beta-\gamma}}{\Gamma(\beta-\gamma+1)} \\
& +\frac{\Gamma(2-\gamma)}{\left|1-\lambda_{2} \mu_{2} \xi^{2(1-\gamma)}\right|}\left[\frac{\left|\lambda_{1}\right|\left(\left|\mu_{1}\right|\left|\lambda_{2}\right| \xi^{1-\gamma}+1\right)}{\left|1-\lambda_{1} \mu_{1}\right|}+\left|\lambda_{2}\right| \xi^{1-\gamma}\right] \frac{1}{\Gamma(\beta-\gamma+1)} \\
& +\frac{\left|\lambda_{1}\right|}{\left|1-\mu_{1} \lambda_{1}\right|} \frac{1}{\Gamma(\beta+1)}, \\
\sigma_{3}= & \frac{\left|\mu_{2}\right| \Gamma(2-\gamma)}{\left|1-\lambda_{2} \mu_{2} \xi^{2(1-\gamma)}\right|}\left[\frac{\left.\left|\mu_{1}\right|\left(\left|\lambda_{1}\right|+\left|\lambda_{2}\right|\right) \xi^{1-\gamma}\right)}{\left|1-\lambda_{1} \mu_{1}\right|}+1\right] \frac{\xi^{\alpha-\gamma}}{\Gamma(\alpha-\gamma+1)} \\
& +\frac{\Gamma(2-\gamma)}{\left|1-\lambda_{2} \mu_{2} \xi^{2(1-\gamma)}\right|}\left[\frac{\left|\mu_{1}\right|\left(\left|\lambda_{1}\right|\left|\mu_{2}\right| \xi^{1-\gamma}+1\right)}{\left|1-\lambda_{1} \mu_{1}\right|}+\left|\mu_{2}\right| \xi^{1-\gamma}\right] \frac{1}{\Gamma(\alpha-\gamma+1)} \\
& +\frac{\left|\mu_{1}\right|}{\left|1-\mu_{1} \lambda_{1}\right|} \frac{1}{\Gamma(\alpha+1)}, \\
\sigma_{4}= & \frac{\left|\lambda_{2}\right| \Gamma(2-\gamma)}{\left|1-\lambda_{2} \mu_{2} \xi^{2(1-\gamma)}\right|}\left[\frac{\left|\mu_{1}\right|\left(\left|\lambda_{1}\right|\left|\mu_{2}\right| \xi^{1-\gamma}+1\right)}{\left|1-\lambda_{1} \mu_{1}\right|}+\left|\mu_{2}\right| \xi^{1-\gamma}\right] \frac{\xi^{\beta-\gamma}}{\Gamma(\beta-\gamma+1)} \\
& +\frac{\Gamma(2-\gamma)}{1-\lambda_{2} \mu_{2} \xi^{2(1-\gamma)}}\left[\frac{\left|\mu_{1}\right|\left(\left|\lambda_{1}\right|+\left|\lambda_{2}\right| \xi^{1-\gamma}\right)}{\mid 1-\lambda_{1} \mu_{1}} \mid+1\right] \frac{1}{\Gamma(\beta-\gamma+1)} \\
& +\left[\frac{\left|\lambda_{1}\right|\left|\mu_{1}\right|}{\left|1-\mu_{1} \lambda_{1}\right|}+1\right] \frac{1}{\Gamma(\beta+1)} \cdot
\end{aligned}
$$

Now we are in a position to present our main results. The methods used to prove the existence and uniqueness solutions of boundary value problem (1)-(2) go via Banach's contraction principle.

\section{Theorem 3.1 Assume that:}

(H1) $f, g:[0,1] \times \mathbb{R} \times \mathbb{R} \rightarrow \mathbb{R}$ are functions and there exist positive constants $l_{1}$ and $l_{2}$ such that, for all $t \in[0,1]$ and $x_{i}, y_{i} \in \mathbb{R}, i=1,2$, we have

$$
\begin{aligned}
& \left|f\left(t, x_{1}, x_{2}\right)-f\left(t, y_{1}, y_{2}\right)\right| \leq l_{1}\left(\left|x_{1}-y_{1}\right|+\left|x_{2}-y_{2}\right|\right), \\
& \left|g\left(t, x_{1}, x_{2}\right)-g\left(t, y_{1}, y_{2}\right)\right| \leq l_{2}\left(\left|x_{1}-y_{1}\right|+\left|x_{2}-y_{2}\right|\right) .
\end{aligned}
$$

If $\left(\sigma_{1}+\sigma_{3}\right) l_{1}+\left(\sigma_{2}+\sigma_{4}\right) l_{2}<1$ then system (1)-(2) has a unique solution on $[0,1]$. 
Proof Define $\sup _{t \in[0,1]} f(t, 0,0)=\rho_{1}<\infty$ and $\sup _{t \in[0,1]} g(t, 0,0)=\rho_{2}<\infty$ and $r>0$ such that

$$
r>\frac{\left(\sigma_{1}+\sigma_{3}\right) \rho_{1}+\left(\sigma_{2}+\sigma_{4}\right) \rho_{2}}{1-\left(\sigma_{1}+\sigma_{3}\right) l_{1}-\left(\sigma_{2}+\sigma_{4}\right) l_{2}} .
$$

We show that $Q\left(B_{r}\right) \subset B_{r}$, where $B_{r}=\{(u, v) \in X \times X:\|(u, v)\| \leq r\}$.

By assumption $(H 1)$, for $(u, v) \in B_{r}, t \in[0,1]$, we have

$$
\begin{aligned}
|f(t, u(t), v(t))| & \leq|f(t, u(t), v(t))-f(t, 0,0)|+|f(t, 0,0)| \\
& \leq l_{1}(|u(t)|+|v(t)|)+\rho_{1} \\
& \leq l_{1}(\|u\|+\|v\|)+\rho_{1} \leq l_{1} r+\rho_{1}
\end{aligned}
$$

and $|g(t, u(t), v(t))| \leq l_{2}(\|u\|+\|v\|)+\rho_{2} \leq l_{2} r+\rho_{2}$, which leads to

$$
\begin{aligned}
& \left|Q_{1}(u, v)(t)\right| \\
& \leq \frac{\left|\mu_{2}\right| \Gamma(2-\gamma)}{\left|1-\lambda_{2} \mu_{2} \xi^{2(1-\gamma)}\right|}\left[\frac{\left|\lambda_{1}\right|\left(\left|\mu_{1}\right|\left|\lambda_{2}\right| \xi^{1-\gamma}+1\right)}{\left|1-\lambda_{1} \mu_{1}\right|}+\left|\lambda_{2}\right| \xi^{1-\gamma}\right] \frac{\xi^{\alpha-\gamma}}{\Gamma(\alpha-\gamma+1)}\left(l_{1} r+\rho_{1}\right) \\
& +\frac{\Gamma(2-\gamma)}{\left|1-\lambda_{2} \mu_{2} \xi^{2(1-\gamma)}\right|}\left[\frac{\left|\lambda_{1}\right|\left(\left|\mu_{1}\right|\left|\lambda_{2}\right| \xi^{1-\gamma}+1\right)}{\left|1-\lambda_{1} \mu_{1}\right|}+\left|\lambda_{2}\right| \xi^{1-\gamma}\right] \frac{1}{\Gamma(\beta-\gamma+1)}\left(l_{2} r+\rho_{2}\right) \\
& +\frac{\Gamma(2-\gamma)}{\left|1-\lambda_{2} \mu_{2} \xi^{2(1-\gamma)}\right|}\left[\frac{\left|\lambda_{1}\right|\left(\left|\mu_{1}\right|+\left|\mu_{2}\right| \xi^{1-\gamma}\right)}{\left|1-\lambda_{1} \mu_{1}\right|}+1\right] \frac{1}{\Gamma(\alpha-\gamma+1)}\left(l_{1} r+\rho_{1}\right) \\
& +\frac{\left|\lambda_{2}\right| \Gamma(2-\gamma)}{\left|1-\lambda_{2} \mu_{2} \xi^{2(1-\gamma)}\right|}\left[\frac{\left|\lambda_{1}\right|\left(\left|\mu_{1}\right|+\left|\mu_{2}\right| \xi^{1-\gamma}\right)}{\left|1-\lambda_{1} \mu_{1}\right|}+1\right] \frac{\xi^{\beta-\gamma}}{\Gamma(\beta-\gamma+1)}\left(l_{2} r+\rho_{2}\right) \\
& +\frac{\left|\lambda_{1}\right|}{\left|1-\mu_{1} \lambda_{1}\right|}\left[\frac{\left|\mu_{1}\right|}{\Gamma(\alpha+1)}\left(l_{1} r+\rho_{1}\right)+\frac{1}{\Gamma(\beta+1)}\left(l_{2} r+\rho_{2}\right)\right]+\frac{1}{\Gamma(\alpha+1)}\left(l_{1} r+\rho_{1}\right) \text {. }
\end{aligned}
$$

Hence,

$$
\left\|Q_{1}(u, v)\right\| \leq\left(\sigma_{1} l_{1}+\sigma_{2} l_{2}\right) r+\sigma_{1} \rho_{1}+\sigma_{2} \rho_{2}
$$

In the same way, we obtain

$$
\left\|Q_{2}(u, v)\right\| \leq\left(\sigma_{3} l_{1}+\sigma_{4} l_{2}\right) r+\sigma_{3} \rho_{1}+\sigma_{4} \rho_{2}
$$

Consequently,

$$
\|Q(u, v)\| \leq\left[\left(\sigma_{1}+\sigma_{3}\right) l_{1}+\left(\sigma_{2}+\sigma_{4}\right) l_{2}\right] r+\left(\sigma_{1}+\sigma_{3}\right) \rho_{1}+\left(\sigma_{2}+\sigma_{4}\right) \rho_{2} \leq r
$$

Now, for $\left(u_{2}, v_{2}\right),\left(u_{1}, v_{1}\right) \in X \times X$ and for any $t \in[0,1]$, we get

$$
\begin{aligned}
& \left|Q_{1}\left(u_{2}, v_{2}\right)(t)-Q_{1}\left(u_{1}, v_{1}\right)(t)\right| \\
& \leq \frac{\left|\mu_{2}\right| \Gamma(2-\gamma)}{\left|1-\lambda_{2} \mu_{2} \xi^{2(1-\gamma)}\right|}\left[\frac{\left|\lambda_{1}\right|\left(\left|\mu_{1}\right|\left|\lambda_{2}\right| \xi^{1-\gamma}+1\right)}{\left|1-\lambda_{1} \mu_{1}\right|}+\left|\lambda_{2}\right| \xi^{1-\gamma}\right] \\
& \quad \times \frac{\xi^{\alpha-\gamma}}{\Gamma(\alpha-\gamma+1)} l_{1}\left(\left\|u_{2}-u_{1}\right\|+\left\|v_{2}-v_{1}\right\|\right)
\end{aligned}
$$




$$
\begin{aligned}
& +\frac{\Gamma(2-\gamma)}{\left|1-\lambda_{2} \mu_{2} \xi^{2(1-\gamma)}\right|}\left[\frac{\left|\lambda_{1}\right|\left(\left|\mu_{1}\right|\left|\lambda_{2}\right| \xi^{1-\gamma}+1\right)}{\left|1-\lambda_{1} \mu_{1}\right|}+\left|\lambda_{2}\right| \xi^{1-\gamma}\right] \\
& \times \frac{1}{\Gamma(\beta-\gamma+1)} l_{2}\left(\left\|u_{2}-u_{1}\right\|+\left\|v_{2}-v_{1}\right\|\right) \\
& +\frac{\Gamma(2-\gamma)}{\left|1-\lambda_{2} \mu_{2} \xi^{2(1-\gamma)}\right|}\left[\frac{\left|\lambda_{1}\right|\left(\left|\mu_{1}\right|+\left|\mu_{2}\right| \xi^{1-\gamma}\right)}{\left|1-\lambda_{1} \mu_{1}\right|}+1\right] \\
& \times \frac{1}{\Gamma(\alpha-\gamma+1)} l_{1}\left(\left\|u_{2}-u_{1}\right\|+\left\|v_{2}-v_{1}\right\|\right) \\
& +\frac{\left|\lambda_{2}\right| \Gamma(2-\gamma)}{\left|1-\lambda_{2} \mu_{2} \xi^{2(1-\gamma)}\right|}\left[\frac{\left|\lambda_{1}\right|\left(\left|\mu_{1}\right|+\left|\mu_{2}\right| \xi^{1-\gamma}\right)}{\left|1-\lambda_{1} \mu_{1}\right|}+1\right] \\
& \times \frac{\xi^{\beta-\gamma}}{\Gamma(\beta-\gamma+1)} l_{2}\left(\left\|u_{2}-u_{1}\right\|+\left\|v_{2}-v_{1}\right\|\right) \\
& +\frac{\left|\lambda_{1}\right|}{\left|1-\mu_{1} \lambda_{1}\right|} \frac{1}{\Gamma(\beta+1)} l_{2}\left(\left\|u_{2}-u_{1}\right\|+\left\|v_{2}-v_{1}\right\|\right) \\
& +\frac{\left|\lambda_{1}\right|\left|\mu_{1}\right|}{\left|1-\mu_{1} \lambda_{1}\right|} \frac{1}{\Gamma(\alpha+1)} l_{1}\left(\left\|u_{2}-u_{1}\right\|+\left\|v_{2}-v_{1}\right\|\right) \\
& +\frac{1}{\Gamma(\alpha+1)} l_{1}\left(\left\|u_{2}-u_{1}\right\|+\left\|v_{2}-v_{1}\right\|\right) \\
& =\left(\sigma_{1} l_{1}+\sigma_{2} l_{2}\right)\left(\left\|u_{2}-u_{1}\right\|+\left\|v_{2}-v_{1}\right\|\right)
\end{aligned}
$$

and consequently we obtain

$$
\left\|Q_{1}\left(u_{2}, v_{2}\right)(t)-Q_{1}\left(u_{1}, v_{1}\right)(t)\right\| \leq\left(\sigma_{1} l_{1}+\sigma_{1} l_{2}\right)\left(\left\|u_{2}-u_{1}\right\|+\left\|v_{2}-v_{1}\right\|\right) .
$$

Similarly,

$$
\left\|Q_{2}\left(u_{2}, v_{2}\right)(t)-Q_{2}\left(u_{1}, v_{1}\right)(t)\right\| \leq\left(\sigma_{3} l_{1}+\sigma_{4} l_{2}\right)\left(\left\|u_{2}-u_{1}\right\|+\left\|v_{2}-v_{1}\right\|\right) .
$$

It follows from (8) and (9) that

$$
\left\|Q\left(u_{2}, v_{2}\right)(t)-Q\left(u_{1}, v_{1}\right)\right\| \leq\left[\left(\sigma_{1}+\sigma_{3}\right) l_{1}+\left(\sigma_{2}+\sigma_{4}\right) l_{2}\right]\left(\left\|u_{2}-u_{1}\right\|+\left\|v_{2}-v_{1}\right\|\right) .
$$

Since $\left(\sigma_{1}+\sigma_{3}\right) l_{1}+\left(\sigma_{2}+\sigma_{4}\right) l_{2}<1, Q$ is a contraction operator. So, by Banach's fixed point theorem, the operator $Q$ has a unique fixed point, which is the unique solution of problem (1)-(2).

The second result is based on the Leray-Schauder alternative.

Lemma 3.2 (Leray-Schauder alternative [18]) Let $F: E \rightarrow E$ be a completely continuous operator (i.e., a map restricted to any bounded set in $E$ is compact). Let

$$
\varepsilon(F)=\{x \in E: x=\lambda F(x) \text { for some } 0<\lambda<1\} .
$$

Then either the set $\varepsilon(F)$ is unbounded or $F$ has at least one fixed point. 


\section{Theorem 3.3 Assume that:}

(H2) $f, g:[0,1] \times \mathbb{R} \times \mathbb{R} \rightarrow \mathbb{R}$ are continuous functions and there exist real constants $k_{i}, \gamma_{i} \geq 0(i=0,1,2)$ and $k_{0}>0, \gamma_{0}>0$ such that $\forall x_{i} \in \mathbb{R}(i=1,2)$, we have

$$
\begin{aligned}
& \left|f\left(t, x_{1}, x_{2}\right)\right| \leq k_{0}+k_{1}\left|x_{1}\right|+k_{2}\left|x_{2}\right|, \\
& \left|g\left(t, x_{1}, x_{2}\right)\right| \leq \gamma_{0}+\gamma_{1}\left|x_{1}\right|+\gamma_{2}\left|x_{2}\right| .
\end{aligned}
$$

If $\left(\sigma_{1}+\sigma_{3}\right) \kappa_{1}+\left(\sigma_{2}+\sigma_{4}\right) \gamma_{1}<1$ and $\left(\sigma_{1}+\sigma_{3}\right) \kappa_{2}+\left(\sigma_{2}+\sigma_{4}\right) \gamma_{2}<1$ then system (1)-(2) has at least one solution on $[0,1]$.

Proof First we show that the operator $Q: X \times X \rightarrow X \times X$ is completely continuous. By the continuity of functions $f$ and $g$, the operator $Q$ is continuous.

Let $\Omega \subset X \times X$ be bounded. Then there exist positive constants $K_{1}$ and $K_{2}$ such that $|f(t, u(t) . v(t))| \leq K_{1},|g(t, u(t) . v(t))| \leq K_{2}, \forall(u, v) \in \Omega$. Then, for any $(u, v) \in \Omega$, we have

$$
\begin{aligned}
& \left|Q_{1}(u, v)(t)\right| \\
& \leq \frac{\left|\mu_{2}\right| \Gamma(2-\gamma)}{\mid 1-\lambda_{2} \mu_{2} \xi^{2(1-\gamma) \mid}}\left[\frac{\left|\lambda_{1}\right|\left(\left|\mu_{1}\right|\left|\lambda_{2}\right| \xi^{1-\gamma}+1\right)}{\left|1-\lambda_{1} \mu_{1}\right|}+\left|\lambda_{2}\right| \xi^{1-\gamma}\right] \frac{\xi^{\alpha-\gamma}}{\Gamma(\alpha-\gamma+1)} K_{1} \\
& \quad+\frac{\Gamma(2-\gamma)}{\left|1-\lambda_{2} \mu_{2} \xi^{2(1-\gamma)}\right|}\left[\frac{\left|\lambda_{1}\right|\left(\left|\mu_{1}\right|\left|\lambda_{2}\right| \xi^{1-\gamma}+1\right)}{\left|1-\lambda_{1} \mu_{1}\right|}+\left|\lambda_{2}\right| \xi^{1-\gamma}\right] \frac{1}{\Gamma(\beta-\gamma+1)} K_{2} \\
& \quad+\frac{\Gamma(2-\gamma)}{\left|1-\lambda_{2} \mu_{2} \xi^{2(1-\gamma)}\right|}\left[\frac{\left|\lambda_{1}\right|\left(\left|\mu_{1}\right|+\left|\mu_{2}\right| \xi^{1-\gamma}\right)}{\left|1-\lambda_{1} \mu_{1}\right|}+1\right] \frac{1}{\Gamma(\alpha-\gamma+1)} K_{1} \\
& \quad+\frac{\left|\lambda_{2}\right| \Gamma(2-\gamma)}{\left|1-\lambda_{2} \mu_{2} \xi^{2(1-\gamma)}\right|}\left[\frac{\left|\lambda_{1}\right|\left(\left|\mu_{1}\right|+\left|\mu_{2}\right| \xi^{1-\gamma}\right)}{\left|1-\lambda_{1} \mu_{1}\right|}+1\right] \frac{\xi^{\beta-\gamma}}{\Gamma(\beta-\gamma+1)} K_{2} \\
& \quad+\frac{\left|\lambda_{1}\right|}{\left|1-\mu_{1} \lambda_{1}\right|}\left[\frac{\left|\mu_{1}\right|}{\Gamma(\alpha+1)} K_{1}+\frac{1}{\Gamma(\beta+1)} K_{2}\right]+\frac{1}{\Gamma(\alpha+1)} K_{1},
\end{aligned}
$$

which implies that $\left\|Q_{1}(u, v)\right\| \leq \sigma_{1} K_{1}+\sigma_{2} K_{2}$. Similarly, we get $\left\|Q_{2}(u, v)\right\| \leq \sigma_{3} K_{1}+\sigma_{4} K_{2}$. Thus, it follows from the above inequalities that the operator $Q$ is uniformly bounded, since $\|Q(u, v)\| \leq\left(\sigma_{1}+\sigma_{3}\right) K_{1}+\left(\sigma_{2}+\sigma_{4}\right) K_{2}$.

Next, we show that $Q$ is equicontinuous. Let $t_{1}, t_{2} \in[0,1]$ with $t_{1}<t_{2}$. Then we have

$$
\begin{aligned}
& \left|Q_{1}\left(u\left(t_{2}\right), v\left(t_{2}\right)\right)-Q_{1}\left(u\left(t_{1}\right), v\left(t_{1}\right)\right)\right| \\
& \leq K_{1} \frac{\xi^{\alpha-\gamma}}{\Gamma(\alpha-\gamma+1)} \frac{\left|\mu_{2}\right|\left|\lambda_{2}\right| \xi^{1-\gamma} \Gamma(2-\gamma)}{\left|1-\lambda_{2} \mu_{2} \xi^{2(1-\gamma)}\right|}\left(t_{2}-t_{1}\right) \\
& \quad+K_{2} \frac{1}{\Gamma(\beta-\gamma+1)} \frac{\left|\lambda_{2}\right| \xi^{1-\gamma} \Gamma(2-\gamma)}{\left|1-\lambda_{2} \mu_{2} \xi^{2(1-\gamma)}\right|}\left(t_{2}-t_{1}\right) \\
& \quad+K_{1} \frac{1}{\Gamma(\alpha-\gamma+1)} \frac{\Gamma(2-\gamma)}{\left|1-\lambda_{2} \mu_{2} \xi^{2(1-\gamma)}\right|}\left(t_{2}-t_{1}\right) \\
& \quad+K_{2} \frac{\xi^{\beta-\gamma}}{\Gamma(\beta-\gamma+1)} \frac{\left|\lambda_{2}\right| \Gamma(2-\gamma)}{\left|1-\lambda_{2} \mu_{2} \xi^{2(1-\gamma)}\right|}\left(t_{2}-t_{1}\right) \\
& \quad+K_{1}\left|\frac{1}{\Gamma(\alpha)} \int_{0}^{t_{2}}\left(t_{2}-s\right)^{\alpha-1} d s-\frac{1}{\Gamma(\alpha)} \int_{0}^{t_{1}}\left(t_{1}-s\right)^{\alpha-1} d s\right| \\
& \leq \\
& K_{1} \frac{\xi^{\alpha-\gamma}}{\Gamma(\alpha-\gamma+1)} \frac{\left|\mu_{2}\right|\left|\lambda_{2}\right| \xi^{1-\gamma} \Gamma(2-\gamma)}{\left|1-\lambda_{2} \mu_{2} \xi^{2(1-\gamma)}\right|}\left(t_{2}-t_{1}\right)
\end{aligned}
$$




$$
\begin{aligned}
& +K_{2} \frac{1}{\Gamma(\beta-\gamma+1)} \frac{\left|\lambda_{2}\right| \xi^{1-\gamma} \Gamma(2-\gamma)}{\left|1-\lambda_{2} \mu_{2} \xi^{2(1-\gamma)}\right|}\left(t_{2}-t_{1}\right) \\
& +K_{1} \frac{1}{\Gamma(\alpha-\gamma+1)} \frac{\Gamma(2-\gamma)}{\left|1-\lambda_{2} \mu_{2} \xi^{2(1-\gamma)}\right|}\left(t_{2}-t_{1}\right) \\
& +K_{2} \frac{\xi^{\beta-\gamma}}{\Gamma(\beta-\gamma+1)} \frac{\left|\lambda_{2}\right| \Gamma(2-\gamma)}{\mid 1-\lambda_{2} \mu_{2} \xi^{2(1-\gamma) \mid}}\left(t_{2}-t_{1}\right) \\
& +K_{1}\left|\int_{t_{1}}^{t_{2}} \frac{\left(t_{2}-s\right)^{\alpha-1}}{\Gamma(\alpha)} d s\right|+K_{1}\left|\int_{0}^{t_{1}} \frac{\left(t_{2}-s\right)^{\alpha-1}-\left(t_{1}-s\right)^{\alpha-1}}{\Gamma(\alpha)} d s\right| \\
& \leq K_{1} \frac{\xi^{\alpha-\gamma}}{\Gamma(\alpha-\gamma+1)} \frac{\left|\mu_{2}\right|\left|\lambda_{2}\right| \xi^{1-\gamma} \Gamma(2-\gamma)}{\left|1-\lambda_{2} \mu_{2} \xi^{2(1-\gamma)}\right|}\left(t_{2}-t_{1}\right) \\
& +K_{2} \frac{1}{\Gamma(\beta-\gamma+1)} \frac{\left|\lambda_{2}\right| \xi^{1-\gamma} \Gamma(2-\gamma)}{\left|1-\lambda_{2} \mu_{2} \xi^{2(1-\gamma)}\right|}\left(t_{2}-t_{1}\right) \\
& +K_{1} \frac{1}{\Gamma(\alpha-\gamma+1)} \frac{\Gamma(2-\gamma)}{\left|1-\lambda_{2} \mu_{2} \xi^{2(1-\gamma)}\right|}\left(t_{2}-t_{1}\right) \\
& +K_{2} \frac{\xi^{\beta-\gamma}}{\Gamma(\beta-\gamma+1)} \frac{\left|\lambda_{2}\right| \Gamma(2-\gamma)}{\left|1-\lambda_{2} \mu_{2} \xi^{2(1-\gamma)}\right|}\left(t_{2}-t_{1}\right)+K_{1} \frac{\left(t_{2}^{\alpha}-t_{1}^{\alpha}\right)}{\Gamma(\alpha+1)}
\end{aligned}
$$

Analogously, we can obtain

$$
\begin{aligned}
& \left|Q_{2}\left(u\left(t_{2}\right), v\left(t_{2}\right)\right)-Q_{2}\left(u\left(t_{1}\right), v\left(t_{1}\right)\right)\right| \\
& \leq K_{1} \frac{\xi^{\alpha-\gamma}}{\Gamma(\alpha-\gamma+1)} \frac{\left|\mu_{2}\right| \Gamma(2-\gamma)}{\left|1-\lambda_{2} \mu_{2} \xi^{2(1-\gamma)}\right|}\left(t_{2}-t_{1}\right) \\
& \quad+K_{2} \frac{1}{\Gamma(\beta-\gamma+1)} \frac{\Gamma(2-\gamma)}{\left|1-\lambda_{2} \mu_{2} \xi^{2(1-\gamma)}\right|}\left(t_{2}-t_{1}\right) \\
& \quad+K_{1} \frac{1}{\Gamma(\alpha-\gamma+1)} \frac{\left|\mu_{2}\right| \xi^{1-\gamma} \Gamma(2-\gamma)}{\left|1-\lambda_{2} \mu_{2} \xi^{2(1-\gamma)}\right|}\left(t_{2}-t_{1}\right) \\
& \quad+K_{2} \frac{\xi^{\beta-\gamma}}{\Gamma(\beta-\gamma+1)} \frac{\left|\mu_{2}\right|\left|\lambda_{2}\right| \xi^{1-\gamma} \Gamma(2-\gamma)}{\left|1-\lambda_{2} \mu_{2} \xi^{2(1-\gamma)}\right|}\left(t_{2}-t_{1}\right)+K_{2} \frac{\left(t_{2}^{\beta}-t_{1}^{\beta}\right)}{\Gamma(\beta+1)} .
\end{aligned}
$$

Then we can easily show that the operator $Q(u, v)$ is equicontinuous. As a consequence of steps together with the Arzela-Ascoli theorem, we find that the operator $Q(u, v)$ is completely continuous.

Finally, it will be verified that the set $\varepsilon=\{(u, v) \in X \times X \mid(u, v)=\lambda Q(u, v), 0 \leq \lambda \leq 1\}$ is bounded. Let $(u, v) \in \varepsilon$, with $(u, v)=\lambda Q(u, v)$. For any $t \in[0,1]$, we have

$$
u(t)=\lambda Q_{1}(u, v)(t), \quad v(t)=\lambda Q_{2}(u, v)(t) .
$$

Then

$$
\begin{aligned}
|u(t)| & \leq \sigma_{1}\left(k_{0}+k_{1}|u|+k_{2}|v|\right)+\sigma_{2}\left(\gamma_{0}+\gamma_{1}|u|+\gamma_{2}|v|\right) \\
& =\sigma_{1} k_{0}+\sigma_{2} \gamma_{0}+\left(\sigma_{1} k_{1}+\sigma_{2} \gamma_{1}\right)|u|+\left(\sigma_{1} k_{2}+\sigma_{2} \gamma_{2}\right)|v|
\end{aligned}
$$

and

$$
\begin{aligned}
|v(t)| & \leq \sigma_{3}\left(k_{0}+k_{1}|u|+k_{2}|v|\right)+\sigma_{4}\left(\gamma_{0}+\gamma_{1}|u|+\gamma_{2}|v|\right) \\
& =\sigma_{3} k_{0}+\sigma_{4} \gamma_{0}+\left(\sigma_{3} k_{1}+\sigma_{4} \gamma_{1}\right)|u|+\left(\sigma_{3} k_{2}+\sigma_{4} \gamma_{2}\right)|v|,
\end{aligned}
$$


which implies

$$
\begin{aligned}
\|u\|+\|v\| \leq & \left(\sigma_{1}+\sigma_{3}\right) k_{0}+\left(\sigma_{2}+\sigma_{4}\right) \gamma_{0}+\left[\left(\sigma_{1}+\sigma_{3}\right) k_{1}+\left(\sigma_{2}+\sigma_{4}\right) \gamma_{1}\right]\|u\| \\
& +\left[\left(\sigma_{1}+\sigma_{3}\right) k_{2}+\left(\sigma_{2}+\sigma_{4}\right) \gamma_{2}\right]\|v\| .
\end{aligned}
$$

Consequently,

$$
\|(u, v)\| \leq \frac{\left(\sigma_{1}+\sigma_{3}\right) k_{0}+\left(\sigma_{2}+\sigma_{4}\right) \gamma_{0}}{\sigma_{0}}
$$

where

$$
\sigma_{0}=\min \left\{1-\left[\left(\sigma_{1}+\sigma_{3}\right) k_{1}+\left(\sigma_{2}+\sigma_{4}\right) \gamma_{1}\right], \quad 1-\left[\left(\sigma_{1}+\sigma_{3}\right) k_{2}+\left(\sigma_{2}+\sigma_{4}\right) \gamma_{2}\right]\right\},
$$

which proves that $\varepsilon$ is bounded. Thus, by Lemma 3.2, the operator $Q$ has at least one fixed point. Hence, the boundary value problem (1)-(2) has at least one solution.

\section{Some examples}

In this section, we will present some examples to illustrate the main results.

Example 1 Consider the following system of fractional boundary value problem:

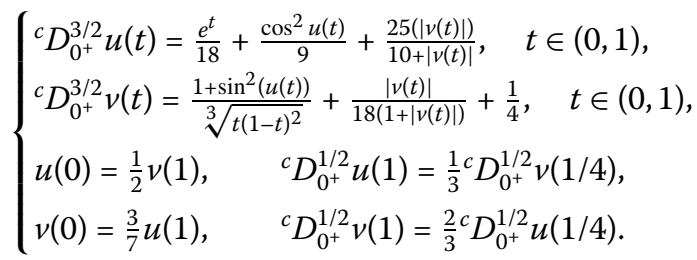

Here $\alpha=\beta=\frac{3}{2}, \gamma=\frac{1}{2}, \lambda_{1}=\frac{1}{2}, \lambda_{2}=\frac{1}{3}, \mu_{1}=\frac{3}{7}, \mu_{2}=\frac{2}{3}, \xi=\frac{1}{4}$. By simple calculation, we found that $\sigma_{1}=2.755358, \sigma_{2}=2.847316, \sigma_{3}=2.10908, \sigma_{4}=2.49716$. Note that

$$
\begin{aligned}
& \left|f\left(t, u_{1}, u_{2}\right)-f\left(t, v_{1}, v_{2}\right)\right| \leq \frac{1}{18}\left|u_{1}-u_{2}\right|+\frac{1}{18}\left|v_{1}-v_{2}\right|, \\
& \left|g\left(t, u_{1}, u_{2}\right)-g\left(t, v_{1}, v_{2}\right)\right| \leq \frac{1}{18}\left|u_{1}-u_{2}\right|+\frac{1}{18}\left|v_{1}-v_{2}\right|,
\end{aligned}
$$

and $\left(\sigma_{1}+\sigma_{3}\right) l_{1}+\left(\sigma_{2}+\sigma_{4}\right) l_{2} \approx 0.56716<1$. Thus all the conditions of Theorem 3.1 are satisfied.

Example 2 Consider the following fractional boundary value problem:

$$
\left\{\begin{array}{l}
{ }^{c} D_{0^{+}}^{3 / 2} u(t)=\frac{1}{6(t+1)^{2}} u(t)+\frac{1}{32} \sin v(t)+1, \quad t \in(0,1), \\
{ }^{c} D_{0^{+}}^{3 / 2} v(t)=\frac{1}{64 \pi} \sin (2 \pi u(t))+\frac{1}{32} v(t)+\frac{1}{2}, \quad t \in(0,1), \\
u(0)=\frac{1}{3} v(1), \quad{ }^{c} D_{0^{+}}^{1 / 3} u(1)=\frac{1}{2} c D_{0^{+}}^{1 / 3} v(1 / 5), \\
v(0)=\frac{3}{5} u(1), \quad{ }^{c} D_{0^{+}}^{1 / 3} v(1)=\frac{2}{5}{ }^{c} D_{0^{+}}^{1 / 3} u(1 / 5) .
\end{array}\right.
$$


Here $\alpha=\beta=\frac{3}{2}, \gamma=\frac{1}{3}, \lambda_{1}=\frac{1}{3}, \lambda_{2}=\frac{1}{2}, \mu_{1}=\frac{3}{5}, \mu_{2}=\frac{2}{5}, \xi=\frac{1}{5}$. Note that

$$
\begin{aligned}
& \left|f\left(t, x_{1}, x_{2}\right)\right| \leq 1+\frac{1}{32}\left|x_{1}\right|+\frac{1}{32}\left|x_{2}\right|, \\
& \left|g\left(t, x_{1}, x_{2}\right)\right| \leq \frac{1}{2}+\frac{1}{32}\left|x_{1}\right|+\frac{1}{32}\left|x_{2}\right| .
\end{aligned}
$$

We get $k_{1}=\frac{1}{32}, k_{2}=\frac{1}{32}, \gamma_{1}=\frac{1}{32}, \gamma_{2}=\frac{1}{32}$. By simple calculation, we found that $\sigma_{1}=2.14850$, $\sigma_{2}=2.1845, \sigma_{3}=2.10890, \sigma_{4}=2.32131$, then we have $\left(\sigma_{1}+\sigma_{3}\right) k_{1}+\left(\sigma_{2}+\sigma_{4}\right) \gamma_{1} \approx 0.27386<1$ and $\left(\sigma_{1}+\sigma_{3}\right) k_{2}+\left(\sigma_{2}+\sigma_{4}\right) \gamma_{2}<1$. By Theorem 3.3, the coupled boundary value problem has at least one positive solution.

\section{Conclusions}

In this work, we have established the existence and uniqueness results for a nonlinear coupled system of Caputo type fractional differential equations supplemented with coupled fractional nonlocal non-separated boundary conditions by using the Banach contraction principle and the Leray-Schauder fixed point theorem. Finally, we give examples to demonstrate our results.

\section{Acknowledgements}

Not applicable.

Funding

Not applicable.

\section{Abbreviations}

Not applicable.

Availability of data and materials

Not applicable.

\section{Competing interests}

The authors declare that they have no competing interests.

Authors' contributions

All authors contributed equally and significantly in writing this article. All authors read and approved the final manuscript.

Authors' information

Meshari Alesemi, Department of Mathematics, Faculty of Science, Jazan University, Jazan, Saudi Arabia. email: malesemi@jazanu.edu.sa

\section{Publisher's Note}

Springer Nature remains neutral with regard to jurisdictional claims in published maps and institutional affiliations.

Received: 24 October 2018 Accepted: 20 February 2019 Published online: 06 March 2019

\section{References}

1. Agarwal, R.P., Ahmad, B., Alsaed, A.: Fractional-order differential equations with anti-periodic boundary conditions: a survey. Bound. Value Probl. 2017, 173 (2017)

2. Agarwal, R.P., Ahmad, B., Garout, D., Alsaedi, A.: Existence results for coupled nonlinear fractional differential equations equipped with nonlocal coupled flux and multi-point boundary conditions. Chaos Solitons Fractals 102, 149-161 (2017)

3. Agarwal, R.P., Alsaedi, A., Alsharif, A., Ahmad, B.: On nonlinear fractional-order boundary value problems with nonlocal multi-point conditions involving Liouville-Caputo derivative. Differ. Equ. Appl. 9, 147-160 (2017)

4. Ahmad, B., Luca, R.: Existence of solutions for a sequential fractional integro-differential system with coupled integral boundary conditions. Chaos Solitons Fractals 104, 378-388 (2017)

5. Ahmad, B., Luca, R.: Existence of solutions for a system of fractional differential equations with coupled nonlocal boundary conditions. Fract. Calc. Appl. Anal. 21(2), 423-441 (2018)

6. Ahmad, B., Nieto, J.J.: Anti-periodic fractional boundary value problems. Comput. Math. Appl. 62, 1150-1156 (2011)

7. Ahmad, B., Nieto, J.J., Alsaedi, A.: Existence and uniqueness of solutions for nonlinear fractional differential equations with non-separated type integral boundary conditions. Acta Math. Sci. 31(6), 2122-2130 (2011) 
8. Ahmad, B., Nieto, J.J., Alsaedi, A., Aqlan, M.: A coupled system of Caputo-type sequential fractional differential equations with coupled (periodic/anti-periodic type) boundary conditions. Mediterr. J. Math. 14(6), Article ID 227 15 pp. (2017)

9. Ahmad, B., Ntouyas, S.K.: Fractional differential inclusions with fractional separated boundary conditions. Fract. Calc Appl. Anal. 15(3), 362-382 (2012)

10. Ahmad, B., Ntouyas, S.K.: Existence results for a coupled system of Caputo type sequential fractional differential equations with nonlocal integral boundary conditions. Appl. Math. Comput. 266, 615-622 (2015)

11. Ahmad, B., Ntouyas, S.K., Alsaed, A., Shammakh, W., Agarwal, R.P.: Existence theory for fractional differential equations with non-separated type nonlocal multi-point and multi-strip boundary conditions. Adv. Differ. Equ. $2018,89(2018)$

12. Ahmad, B., Ntouyas, S.K., Alsaedi, A.: On a coupled system of fractional differential equations with coupled nonlocal and integral boundary conditions. Chaos Solitons Fractals 83, 234-241 (2016)

13. Alsulami, H.H., Ntouyas, S.K., Agarwal, R.P., Ahmad, B., Alsaedi, A.: A study of fractional-order coupled systems with a new concept of coupled non-separated boundary conditions. Bound. Value Probl. 2017, 68 (2017)

14. Baleanu, D., Diethelm, K., Scalas, E., Trujillo, J.J.: Fractional Calculus Models and Numerical Methods. Series on Complexity, Nonlinearity and Chaos. World Scientific, Boston (2012)

15. Das, S.: Functional Fractional Calculus for System Identification and Control. Springer, New York (2008)

16. Delbosco, D.: Fractional calculus and function spaces. J. Fract. Calc. 6, 45-53 (1994)

17. Goodrich, C.: Existence and uniqueness of solutions to a fractional difference equation with nonlocal conditions. Comput. Math. Appl. 61, 191-202 (2011)

18. Granas, A., Dugundji, J.: Fixed Point Theory. Springer, New York (2005)

19. Henderson, J., Kosmatov, N.: Eigenvalue comparison for fractional boundary value problems with the Caputo derivative. Fract. Calc. Appl. Anal. 17, 872-880 (2014)

20. Henderson, J., Luca, R.: Positive solutions for a system of semipositone coupled fractional boundary value problems. Bound. Value Probl. 2016, 61 (2016)

21. Henderson, J., Luca, R., Tudorache, A.: On a system of fractional differential equations with coupled integral boundary conditions. Fract. Calc. Appl. Anal. 18(2), 361-386 (2015)

22. Kilbas, A.A., Srivastava, H.M., Trujillo, J.J.: Theory and Applications of Fractional Differential Equations. Elsevier, Amsterdam (2006)

23. Lazarevic, M.P., Spasic, A.M.: Finite-time stability analysis of fractional order time-delay systems: Gronwall's approach. Math. Comput. Model. 49, 475-481 (2009)

24. Liu, X., Liu, Y.: Fractional differential equations with fractional non-separted boundary conditions. Electron. J. Differ. Equ. 2013(25), 1 (2013)

25. Liu, Z.H., Sun, J.H.: Nonlinear boundary value problems of fractional differential systems. Comput. Math. Appl. 64(4)، 463-475 (2012)

26. LV, L., Wang, J.R., Wei, W.: Existence and uniqueness results for fractional differential equations with boundary value conditions. Opusc. Math. 31(4), 629-643 (2011)

27. Miller, K.S., Ross, B.: An Introduction to the Fractional Calculus and Fractional Differential Equations. Wiley, New York (1993)

28. Ntouyas, S.K., Tariboon, J.: Fractional boundary value problems with multiple orders of fractional derivatives and integrals. Electron. J. Differ. Equ. 2017, 100 (2017)

29. Podlubny, l.: Fractional Differential Equations. Academic Press, New York (1999)

30. Qarout, D., Ahmad, B., Alsaedi, A.: Existence theorems for semi-linear Caputo fractional differential equations with nonlocal discrete and integral boundary conditions. Fract. Calc. Appl. Anal. 19, 463-479 (2016)

31. Rao, S.N.: Multiple positive solutions for a system of Riemann-Liouville fractional order two point boundary value problems. Panam. Math. J. 25(1), 66-81 (2015)

32. Rao, S.N.: Existence and multiplicity for a system of fractional higher-order two-point boundary value problem. J. Appl. Math. Comput. 51, 93-107 (2016)

33. Rao, S.N., Zico, M.M.: Positive solutions for a coupled system of nonlinear semipositone fractional boundary value problems. Int. J. Differ. Equ. 2019, Article ID 2893857, 9 pp. (2019)

34. Sudsutad, W., Tariboon, J.: Boundary value problems for fractional differential equations with three-point fractional integral boundary conditions. Adv. Differ. Equ. 2012, 93 (2012)

35. Yuan, C., Jiang, D., O'Regan, D., Agarwal, R.P.: Multiple positive solutions to systems of nonlinear semipositone fractional differential equations with coupled boundary conditions. Electron. J. Qual. Theory Differ. Equ. 13, 1 (2012)

36. Zhai, C., Xu, L.: Properties of positive solutions to a class of four-point boundary value problem of Caputo fractional differential equations with a parameter. Commun. Nonlinear Sci. Numer. Simul. 19, 2820-2827 (2014)

37. Zhou, Y.: Basic Theory of Fractional Differential Equations. World Scientific, Hackensack (2014) 